\title{
Cristallisation et orientation induites du PET sous sollicitations biaxiales
}

\author{
Yann Marco ${ }^{a}$, Luc Chevalier et Mohend Chaouche \\ LMT-Cachan, 61 avenue du Président Wilson, 94235 Cachan Cedex, France
}

Reçu le 10 juin 2003, accepté le 7 novembre 2003

\begin{abstract}
Résumé - Au cours des procédés de fabrication utilisant le PET (Poly-éthylène Téréphthalate) les déformations biaxiales vécues par le matériau induisent des changements importants de sa microstructure. La compréhension de ces modifications est cruciale pour permettre la mise au point de modèles prédictifs des caractéristiques mécaniques finales. Afin d'identifier les effets d'une sollicitation thermomécanique complexe sur la microstructure (cristallinité, orientation moléculaire, taille des zones cristallines), des essais de traction biaxiale, à chaud, ont été réalisées sur des éprouvettes amorphes. La microstructure du matériau a ensuite été étudiée par densimétrie, dichroïsme infra-rouge et diffraction X. Les influences du taux, de la vitesse et de la température d'élongation ainsi que du séquençage ont été étudiées.
\end{abstract}

Mots clés : PET / multiaxial / cristallisation induite / microstructure

Abstract - Induced crystallization and orientation in PET under biaxial strain state. During the industrial process using PET, the biaxial strain paths experienced by the material leads to important microstructure changes. The understanding of these modifications is very important in order to define models able to predict the final product mechanical strength. In order to identify the effects of a complex thermo-mechanical loading on the induced microstructure (including the crystalline ratio, the molecular orientation and the crystallite size), PET injection-molded specimens were subjected to biaxial elongation tests. The microstructure of the specimens was then investigated using differential densimetry, IR dichroïsm and WAXD using a synchrotron beam. Influences of strain rate, temperature and strain path sequence on crystallites size and orientation are evaluated.

Key words: PET / multiaxial / induced crystallization / microstructure

\section{Introduction}

Le Poly(-éthylène téréphthalate) (PET) est très largement utilisé à l'état semi-cristallin dans l'industrie (bouteilles, fibres textiles, films, ...). Les origines de ce succès sont multiples : le matériau en lui-même présente de bonnes propriétés optiques et chimiques (imperméabilité au $\mathrm{CO}_{2}$ par exemple), mais surtout il a la capacité de se modifier au fur et à mesure de sa déformation. Ces modifications de la microstructure facilitent sa mise en forme, d'une part, et augmentent considérablement ses propriétés mécaniques, d'autre part. Les propriétés mécaniques du produit final dépendent ainsi essentiellement de la texture microstructurale (orientation et extension des chaînes) et du taux de cristallinité du matériau. Ces paramètres sont très influencés par l'histoire thermique et par la déformation permanente induite par le procédé. Dès lors, la compréhension des relations

\footnotetext{
a Auteur correspondant : yann.marco@univ-rennes1.fr
}

entre les paramètres de la mise en forme (déformation, température, géométrie, vitesse...) et la microstructure induite est cruciale afin de mieux prévoir les propriétés mécaniques et donc la tenue en service du produit fini.

Notre étude se base sur l'exemple du procédé de soufflage des bouteilles plastiques, dont la description détaillée est disponible dans un numéro précédent [1]. Ce procédé comprend deux étapes principales : l'injection d'une préforme puis son chauffage entre 10 et $30^{\circ} \mathrm{C}$ au dessus de la température de transition vitreuse du PET (environ $80^{\circ} \mathrm{C}$ ) et enfin sa déformation par étiragesoufflage dans un moule. La figure 1 met en parallèle les différentes étapes du procédé et les microstructures associées.

La prédiction de l'écoulement cinématique de la préforme et celle des caractéristiques géométriques finales de la bouteille (distribution des épaisseurs, évolution des rayons) est relativement bien maitrisée depuis les premières études sur ce procédé (Kamal, Cakmak $[2,3]$ ), mais, et ce malgré des études plus rhéologiques sur 


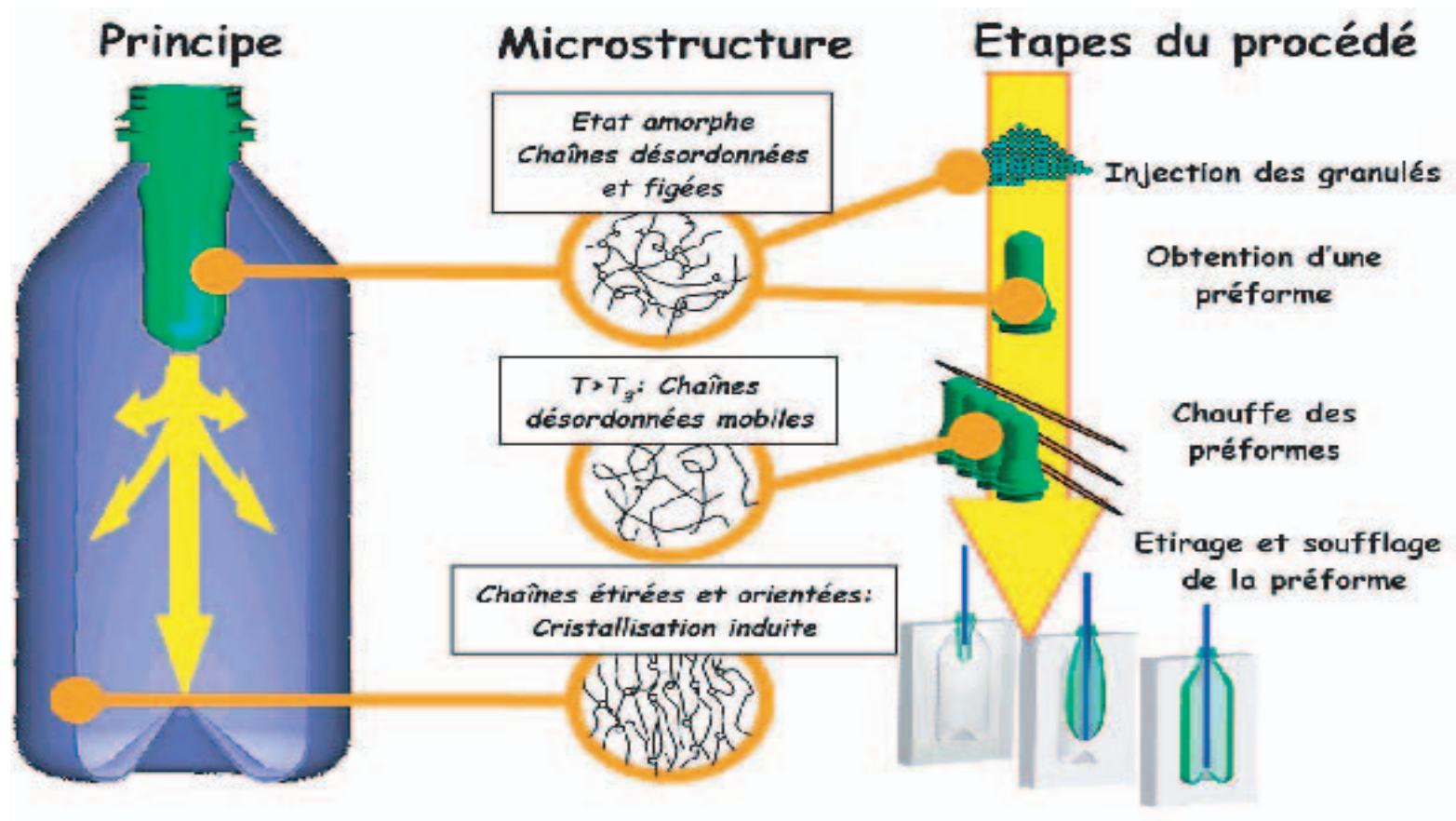

Fig. 1. Principales étapes du procédé et évolution de la microstructure.

le comportement du matériau en étirage (Axtell, Vigny [4,5]), l'influence de l'écoulement du matériau sur ses caractéristiques mécaniques et donc celles de la bouteille reste difficile à modéliser. Réciproquement, les modifications de la microstructure influent aussi sur le comportement du matériau au cours de l'étirage. Une étude microstructurelle du matériau est alors indispensable afin de mieux comprendre ce couplage complexe. Des observations ex situ ou in situ de la morphologie, de la microstructure et de la cristallisation du PET (Casey, Mahendrasingam, Gorlier [6-8]) sont ainsi réalisées, permettant d'étudier l'influence de nombreux paramètres et de mettre en évidence, notamment, le début de la cristallisation induite par l'étirage.

Ces dernières études restent essentiellement uniaxiales. Cependant, au cours de sa mise en forme par un procédé industriel tel que l'étirage-soufflage, le polymère est soumis à des sollicitations beaucoup plus complexes et, du fait de la forte anisotropie des chaînes constituant le matériau polymère, les types de déformations imposés sont cruciaux pour la microstructure induite. Ainsi, Mc Kerron et al. [9] ont montré que la microstructure d'une bouteille est très hétérogène suivant la zone d'étude mais aussi dans son épaisseur. Le but de notre étude est de considérer l'effet d'un champ de déformation complexe sur la microstrucure générée. Afin de nous abstraire des hétérogénéités liées aux essais de caractérisation proches d'un procédé de fabrication, nous avons mis en place notre propre montage de traction multi-axial. Celuici nous permet par ailleurs de déformer des éprouvettes massives, d'épaisseur identique à celle des préformes utilisées en soufflage. La microstructure du matériau est ici caractérisée par mesures ex situ par diffraction des rayons $\mathrm{X}$.

\section{Outils expérimentaux}

\subsection{Montage de chauffage par rayonnement infra-rouge}

Le PET, comme la plupart des polymères, conduit mal la chaleur. Les techniques de chauffage par convection et conduction peuvent imposer des temps de chauffage important, induisant une cristallisation thermique à proscrire dans notre étude. Par ailleurs, ces techniques peuvent induire des hétérogénéités microstructurelles entre la peau et le cour de l'éprouvette. Afin d'éviter ces désagréments, nous avons mis au point un montage de chauffage par infra-rouge, à l'image des solutions adoptées industriellement, qui nous permet de chauffer rapidement et de manière homogène dans la masse. De plus, cette solution permet de laisser l'une des faces visible pour la caméra, et, avantage appréciable, les mors n'étant pas transparents, nous ne chauffons que la zone utile, évitant ainsi l'amincissement et le glissement dans les mors.

\subsection{Mesure du champ de déformation}

Les champs de déformations obtenus au cours des essais sont non homogènes et nécessitent une démarche d'observation des champs de déplacement au fur et à mesure de la déformation. Pour cela, l'une des faces de l'éprouvette est peinte en noir puis un mouchetis blanc est déposé. L'évolution du mouchetis est suivie par une caméra CCD puis analysée par un logiciel d'inter-corrélation d'images développé au laboratoire (Correli $\left.{ }^{\mathrm{LMT}},[10]\right)$ et validé en grandes déformations sur 


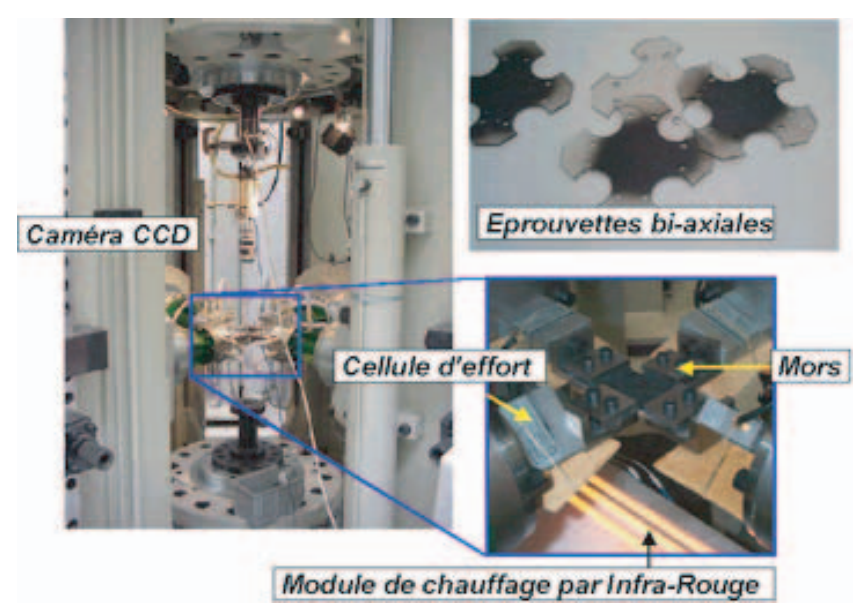

Fig. 2. Vue générale du montage expérimental.

les élastomères [11]. Des résultats typiques obtenus par cette technique sont présentés figure 6 .

\subsection{Montage de sollicitation multi-axiale}

Des essais biaxiaux ont été réalisés sur la machine ASTREE en utilisant des programmes de pilotage et d'acquisition programmés sous LabVIEW ${ }^{\circledR}$. Le matériau est un PET (99 21W EASTMANN) utilisé pour l'étiragesoufflage. Les éprouvettes sont injectées de manière à obtenir un matériau initialement amorphe, puis usinées en forme de croix. La figure 2 présente les différents éléments de ces essais.

\section{Essais multi-axiaux}

\subsection{Essais simultanés et séquencés}

Deux types d'essais biaxiaux ont été réalisés pour différentes températures $\left(90\right.$ et $\left.100{ }^{\circ} \mathrm{C}\right)$, vitesses $(8,20$ et $40 \mathrm{~mm} . \mathrm{s}^{-1}$ ) et taux d'élongation. Au cours du premier essai (Fig. 3), l'éprouvette est étirée de manière simultanée dans des directions orthogonales. Dans le second (Fig. 4), l'éprouvette est étirée d'abord suivant une direction puis suivant l'autre. Les taux d'étirages dans les deux directions étant les mêmes pour ces deux types d'essais. Pour chaque sollicitation, l'éprouvette est portée à température, étirée, puis relaxe dans la configuration étirée.

\subsection{Résultats mécaniques des essais}

La figure 5 illustre que, comme en traction uniaxiale, les essais biaxiaux présentent un comportement durcissant qui montre les mêmes sensibilités à la température et à la vitesse d'élongation.

Ces essais sont hétérogènes et une analyse complète est en cours, utilisant la comparaison à des simulations numériques, comme cela a été fait sur des élastomères [12]. Il est cependant possible d'évaluer le comportement du PET pour les tests de traction biaxiale simultanée, comme l'illustre la figure 6 . En considérant que la déformation suivant la diagonale est uniforme (vérification à l'aide des mesures de champ) et en supposant l'incompressibilité du matériau, on en déduit la contrainte de Cauchy à partir de la force de traction $\mathbf{F}$ mesurée sur un bras, de l'épaisseur e (liée à l'élongation mesurée) et de la longueur L de la diagonale.

On peut dès lors comparer les comportements uniaxiaux et biaxiaux du PET, ce qui confirme qu'à conditions d'étirage identiques, la contrainte uniaxiale est presque deux fois plus faible que celle constatée sous sollicitation biaxiale.

\section{Changements microstructuraux}

\subsection{Cristallisation induite}

Le premier indice global de l'évolution de la microstructure est le taux de cristallinité qui peut être relié aux paramètres thermomécaniques de l'étirage : des échantillons sont découpés dans la zone centrale des éprouvettes puis le taux de cristallinité est mesuré par densimétrie. La figure 7 présente les résultats obtenus. Sur la figure en bas à droite, on montre que la cristallisation ne se produit qu'au dessus d'un taux d'étirage critique, typiquement de l'ordre de $\lambda=2$. Ce taux est proche de celui qu'on observe pour un étirage uniaxial. C'est aussi l'étirage correspondant à un point d'inflexion sur les courbes de comportement et au début du durcissement. Le graphique en bas à gauche montre l'influence de la vitesse d'étirage, à élongation forte $(\lambda \approx 3,5)$ : la cristallinité finale augmente avec la vitesse, jusqu'à saturer à un taux ultime (de l'ordre de $45 \%$ ). Dans le graphique du haut, à gauche, l'influence de la température est illustrée pour deux essais biaxiaux séquencés. On trouve également ce résultat pour les essais simultanés : à mesure que la température augmente, le taux de cristallinité final diminue. Il est vraisemblable que, tout comme pour un étirage uniaxial, ce résultat devienne moins vrai pour des vitesses de déformation plus importantes. Enfin, le graphique en haut à droite compare un essai biaxial simultané à l'essai séquencé. Il semble que le premier étirage dans la direction 1 facilite la cristallisation au terme du second étirage dans la direction orthogonale. Ce résultat reste à confirmer car il est délicat d'obtenir exactement les mêmes états de déformations pour les essais simultanés et séquencés. La mesure du taux de cristallinité donne une bonne indication globale de l'évolution de la microstructure, mais ne permet pas d'avoir accès ni à la taille des cristaux, ni à l'orientation des chaînes moléculaires. Par ailleurs, elle résulte à la fois de la cristallisation apparue au cours de l'étirage et de celle apparue durant la relaxation sous contraintes, on peut donc la relier aux propriétés finales du matériau mais sa contribution au durcissement du matériau ne peut en être déduite directement. 

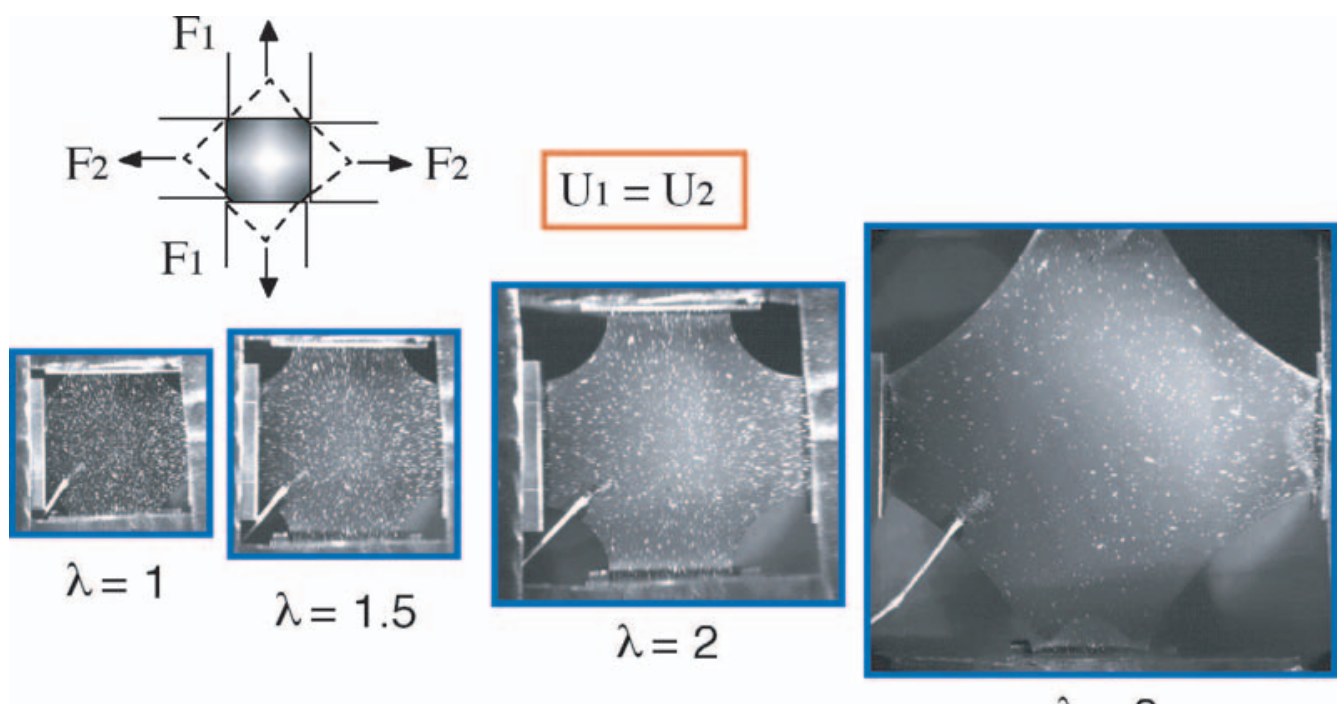

$\lambda=3$

Fig. 3. Principe de l'essai de traction simultanée.
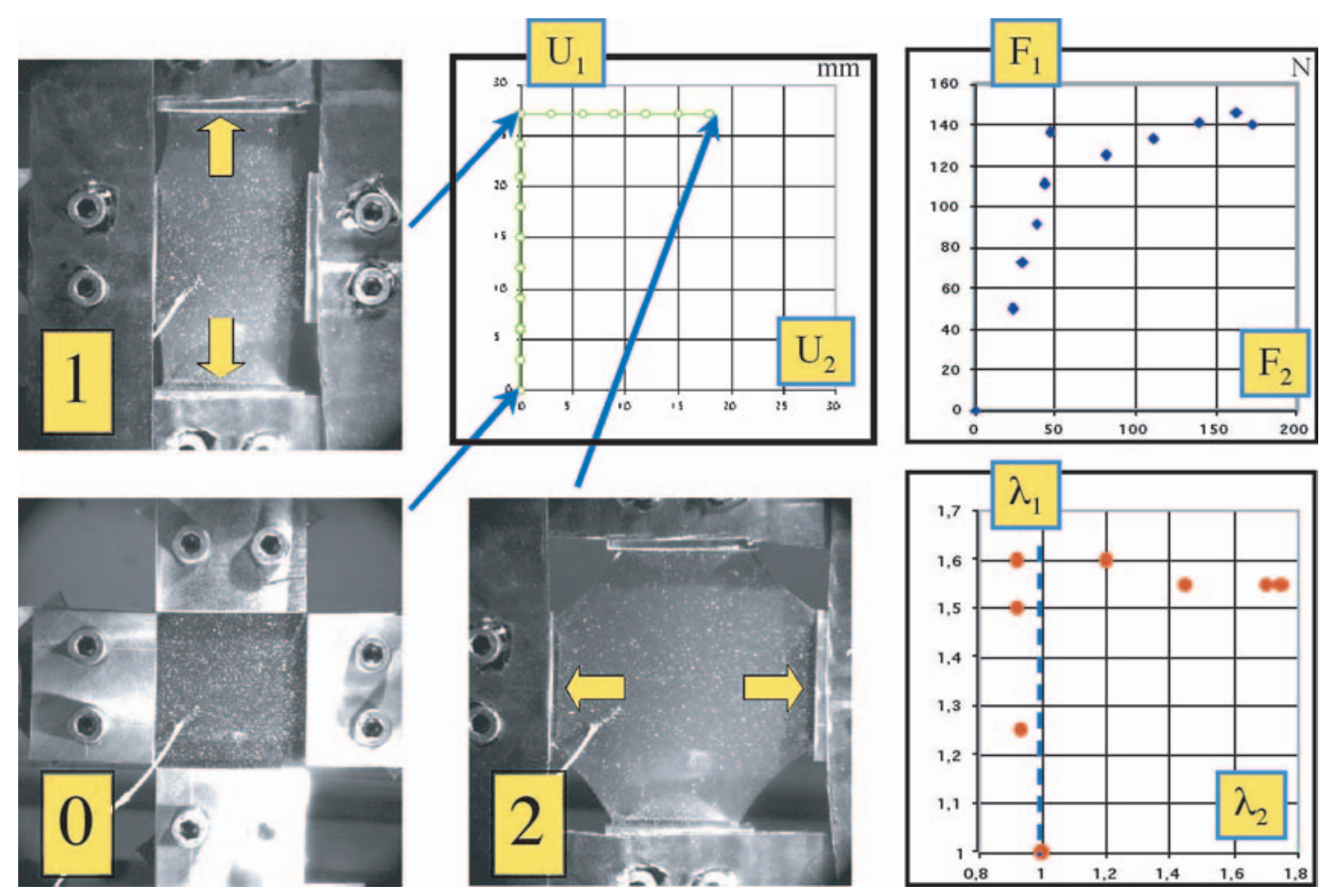

Fig. 4. Principe de l'essai de traction séquencée.

\subsection{Analyse par diffraction $X$}

La diffraction des rayons X (ici aux grands angles : WAXD) permet d'avoir accès à la fois à l'orientation des chaînes, mais aussi à la taille des cristallites. Le rayonnement synchrotron est généralement réservé aux mesures en cours d'essais, la puissance importante du faisceau permettant des temps d'exposition courts. Son usage se justifie ici car il permet d'éviter l'atténuation du faisceau malgré des éprouvettes massives et de mesurer des tailles de cristaux faibles.
Les mesures ont été réalisées sur la ligne D43 du LURE (ORSAY). Les éprouvettes sont scannées par un faisceau de longueur d'onde $1,45 \AA$ (soit $0,145 \mathrm{~nm}$ ) et de diamètre $0,5 \mathrm{~mm}$. La distance entre le faisceau et l'échantillon étant de $80 \mathrm{~mm}$. La figure 8 explique comment évaluer qualitativement les clichés obtenus qui sont du type Debye-Scherrer.

Pour plus de précisions sur les techniques de dépouillement utilisées pour déterminer l'orientation et la taille de cristallites, une explication complète est disponible dans un article récent [13]. La figure 9 présente 

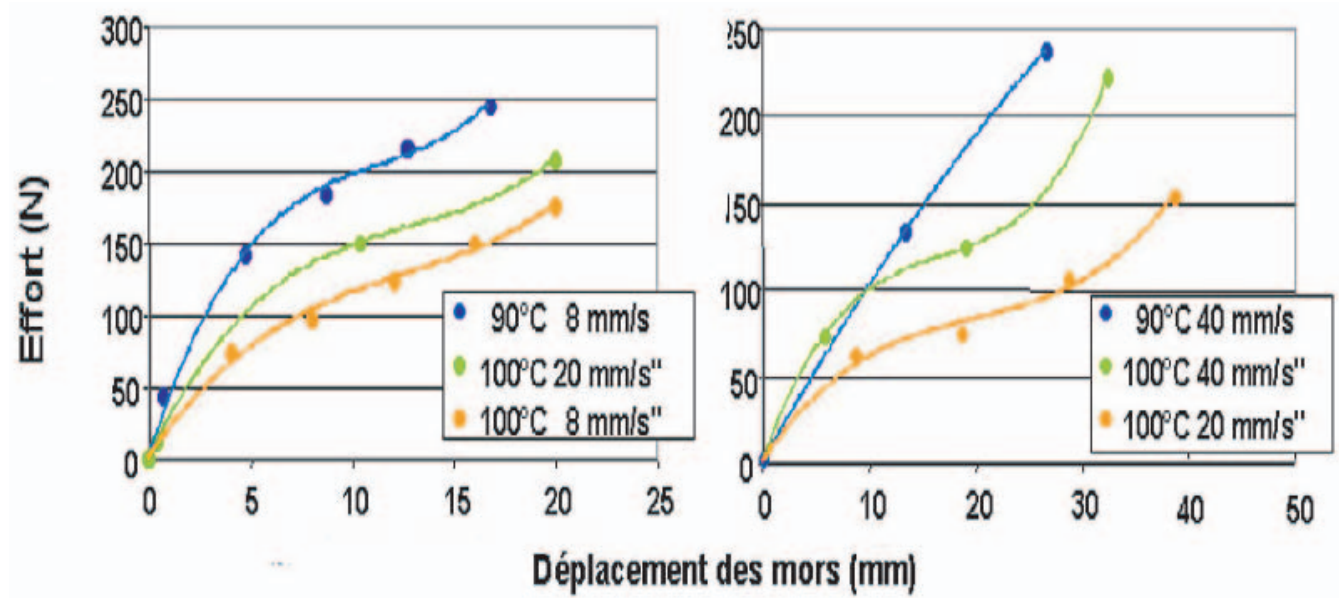

Fig. 5. Courbes efforts déplacements.
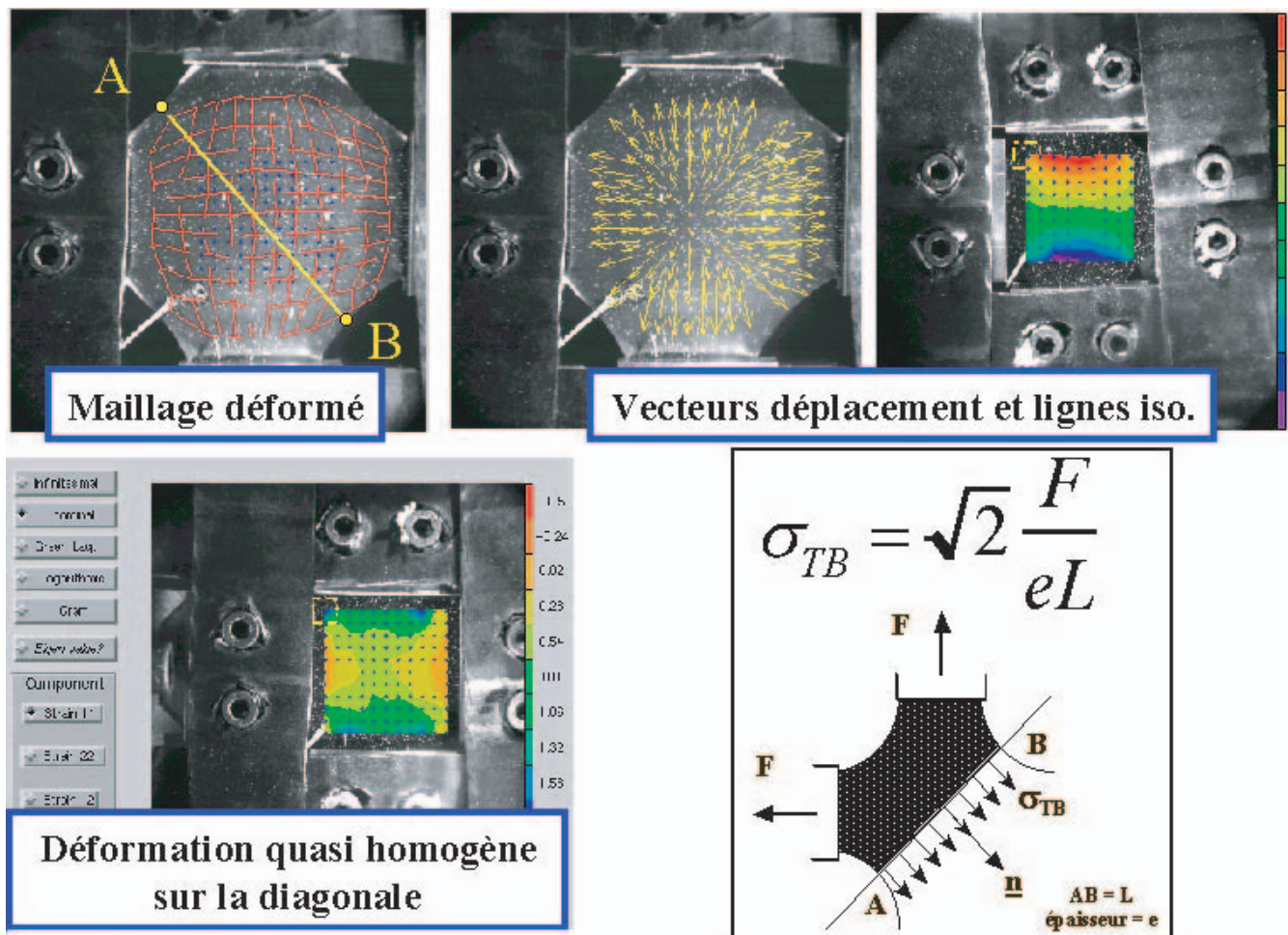

Vecteurs déplacement et lignes iso.

Fig. 6. Dépouillement analytique.

un cliché typique de diffraction obtenu par WAXD ainsi que les reflections cristallographiques associées en utilisant la cellule élémentaire du PET décrite par Daubeny et al. [14]. Nous retrouvons sur cette figure les familles de plans cristallographiques qui nous intéressent et qui sont les plans $(-105)$, dont la normale est représentative de la direction des chaînes, les plans (100) dont la normale est proche de celle des noyaux benzéniques, et les plans (010) qui nous donnent une indication de l'épaisseur des lamelles cristallines.

Notons par ailleurs que pour déterminer complètement l'orientation des cristaux dans le matériau, il faudrait obtenir et dépouiller ce type de cliché dans trois directions orthogonales. Le faisceau $\mathrm{X}$ est ici maintenu orthogonal à la surface de l'échantillon car nous faisons l'hypothèse (vérifiée par de nombreux auteurs) que les plaquettes cristallines s'orientent dans le plan de la traction et ne se désorientent quasiment pas durant la relaxation.

\subsection{Orientation induite}

La figure 10 illustre la correspondance entre un champ de déformations hétérogène mesuré par inter-corrélation d'images et les microstructures mesurées dans différentes 

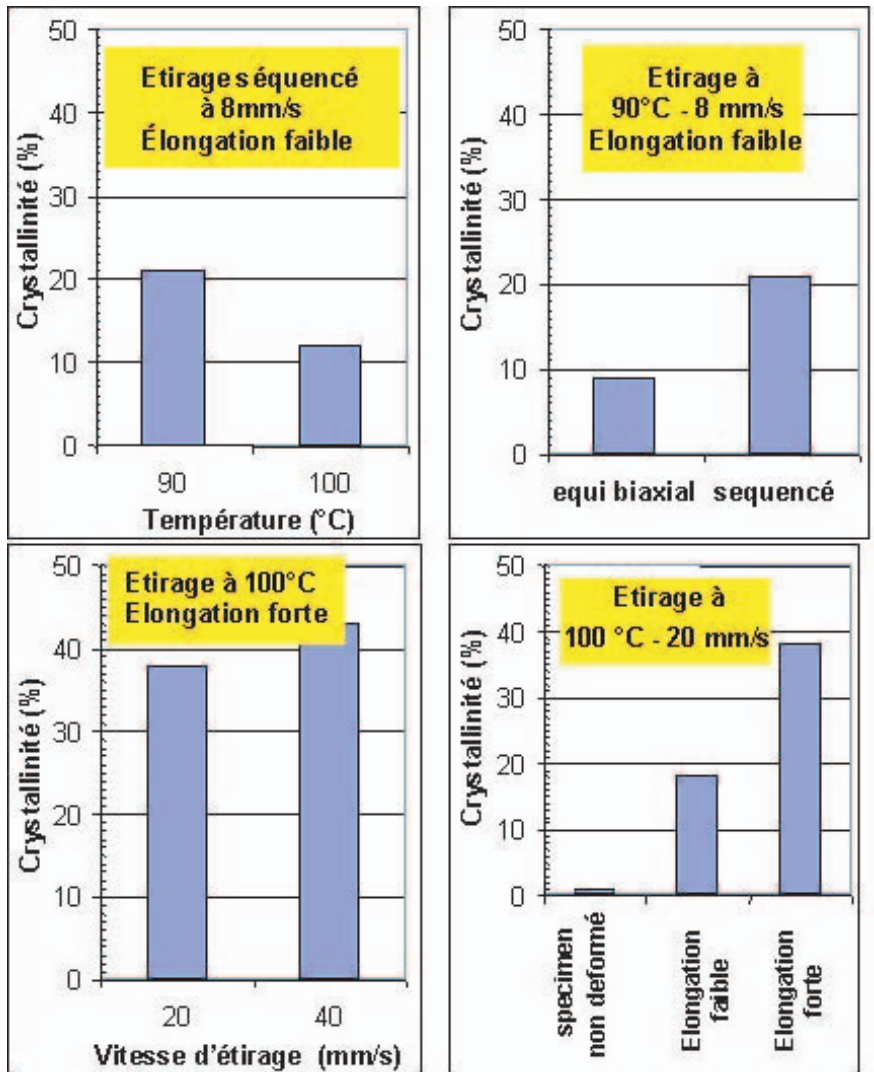

Fig. 7. Influence des conditions d'étirage sur le taux de cristallinité.

zones, pour une éprouvette étirée simultanément. Comme on peut s'y attendre, les chaînes sont très fortement orientées suivant les directions de traction dans les zones « $1 »$ et $\ll 2 »$ (voir Fig. 10), où le champ de déformation mesuré est proche de celui d'une traction plane. Dans la zone centrale, où la déformation est quasiment équilibrée on ne constate aucune orientation préférentielle. Une légère différence entre les déformations suivant l'une ou l'autre des directions peut néanmoins suffire à donner une orientation à la microstructure. Dans la zone de la diagonale, les chaînes sont fortement orientées à $45^{\circ}$ des directions de traction, ce qui est cohérent avec une déformation tendant vers l'uniaxial en se rapprochant du bord libre.

Les orientations mesurées sont de fait très proches des directions principales des contraintes (reliées au tenseur de conformation des chaînes) attendues pour ce type d'essai. Ainsi, comme dans des parutions récentes $[9,15,16]$ pour des mesures aussi bien in que ex situ, la relaxation n'a que peu d'influence sur l'orientation des chaînes. Ce résultat est également confirmé par la faible déviation (environ $10^{\circ}$ ) des réflections équatoriales. Cela peut être expliqué par la cristallisation induite qui empêcherait la relaxation des chaînes. Le taux de cristallinité élevé mesuré soutient cette hypothèse. En effet, même si la cristallisation s'est produite en partie durant la relaxation des contraintes, la cinétique de cristallisation sous contraintes (pour les conditions de vitesse et de température de nos essais) est beaucoup plus rapide que les temps de relaxation des chaînes. De plus, une étude récente par Mahendrasingam et al. [16] montre que ces mêmes conditions devraient permettre un début de cristallisation en cours d'étirage, ce qui confirme le blocage de l'orientation donnée aux chaînes par la déformation locale.

La figure 11 illustre comment les paramètres expérimentaux (vitesse de déformation, élongation, température, trajets de chargement) peuvent influencer les figures de diffraction. Nous retrouvons sur ces figures le comportement décrit pour le taux de cristallinité : l'existence d'une élongation critique et les influences opposées de la vitesse et de la température d'étirage, qui sont aussi clairement observées par des mesures in situ [17]. On peut observer par ailleurs, que pour les essais de traction séquencés, les cristaux restent principalement orientés suivant la première direction de traction, comme cela a aussi été observé pour les films [18]. C'est probablement parce que l'élongation suivant la seconde direction n'est pas suffisamment importante, ni pour casser les cristaux existants, ni pour modifier la première orientation. Ces mesures réalisées par diffraction $\mathrm{X}$ ont été corrélées lors d'observations par dichroïsme infra-rouge [19].

\subsection{Taille des cristallites}

La connaissance de la taille des cristaux suivant trois directions indépendantes permet d'évaluer le volume de ceux-ci. La figure 12 présente la variation du volume des cristallites pour différentes conditions expérimentales. Nous nous sommes intéressés aux microstructures correspondant à la zone centrale et à celles proches des mors où le champ de déformation est proche de la traction plane (voir Fig. 10).

Pour des élongations faibles, les cristallites restent petites aux 2 températures testées, et ce quelle que soit la vitesse d'étirage. On retrouve les influences concurrentes de la vitesse et de la température d'étirage. Ces tendances peuvent être constatées à la fois dans la zone centrale et dans les zones des bras de l'éprouvette. Lorsque l'élongation dépasse un seuil critique $(\lambda \approx 2)$, le volume des cristallites augmente de manière considérable. Cela est dû essentiellement à une croissance importante des lamelles cristallines suivant l'axe des chaînes. Cet effet est d'autant plus fort que la vitesse d'étirage est élevée, mais la taille des cristaux suivant la direction (100) semble elle aussi sensible à l'élongation. On peut également noter que la croissance des lamelles suivant l'axe des chaînes est plus importante dans les zones de «traction plane» que dans celle de traction équi-biaxiale. Des essais uniaxiaux illustrent également ce phénomène de croissance anisotrope suivant la direction de traction. La dimension la plus faible des lamelles est orientée suivant la direction (010), cette «épaisseur » est presque indépendante de l'élongation et de la vitesse d'étirage. Les valeurs obtenues pour les deux zones d'étude sont proches et aucune influence du mode de sollicitation n'a pu être mise en évidence.

Par comparaison du taux de cristallinité avec le volume des cristallites, il est possible d'évaluer le nombre 

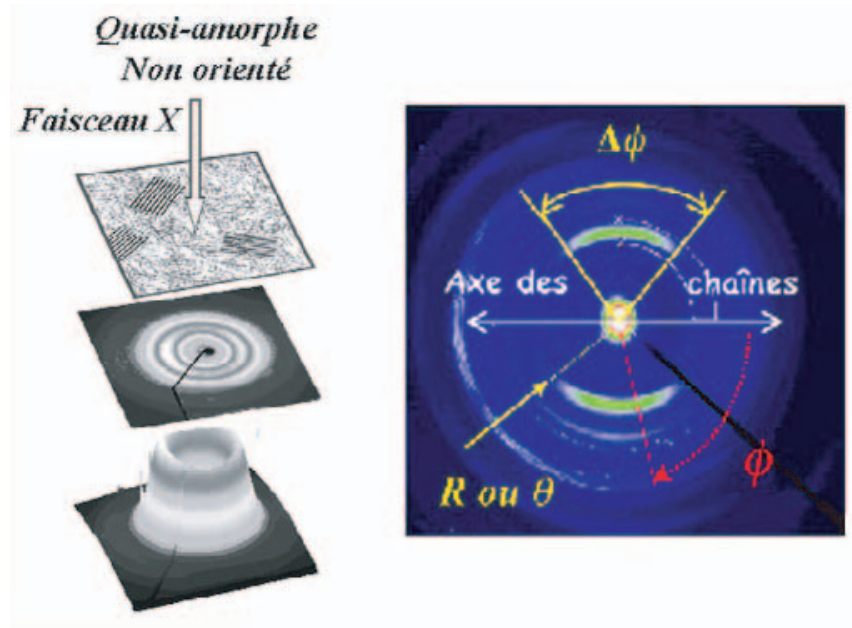

Cristallisé et orienté

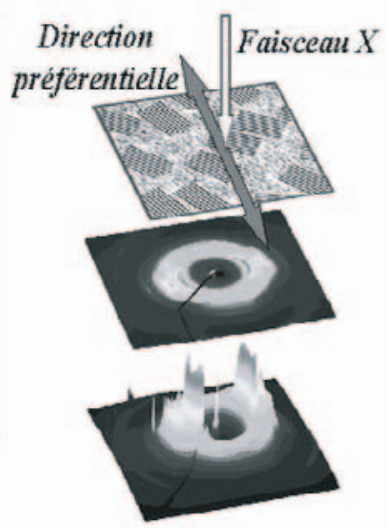

Fig. 8. Clichés typiques et éléments rudimentaires d'analyse : plus $\Delta \phi$ est petit et plus la proportion de chaînes orientées est grande (un échantillon non orienté donne un anneau continu); plus l'épaisseur suivant $\mathbf{R}$ est faible et plus les cristallites sont grands (un échantillon amorphe donne une seule tache épaisse).

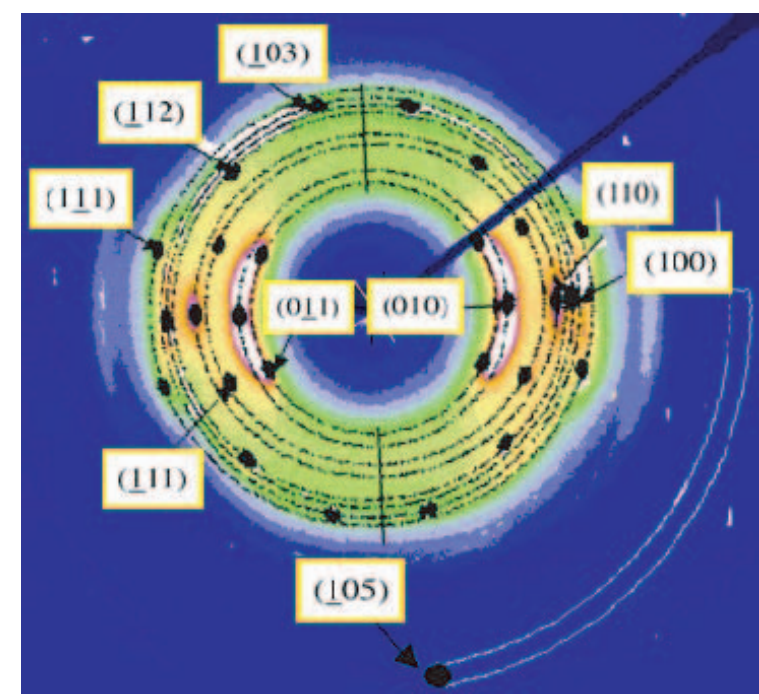

Fig. 9. Cliché et pics cristallographiques associés.

moyen de cristallites par unité de volume [5]. On illustre ainsi l'importance du type de déformation et de leur ordre d'application. Par exemple, les tests de traction séquencée conduisent à des cristaux plus petits et plus nombreux que les essais de bitraction simultanée, en particulier si l'on compare les résultats dans la zone centrale. Cette observation illustre la destruction des blocs cristallins générés lors de l'étirage suivant la première direction, lorsque l'on tire dans la direction orthogonale. Et ce même si, comme nous l'avons vu plus haut, le taux d'étirage suivant la deuxième direction semble trop faible pour réorienter les cristaux suivant cette nouvelle direction de traction. Notons que pour des élongations élevées, l'augmentation de la vitesse d'étirage conduit à des lamelles cristallines plus importantes (et surtout plus longues) mais aussi moins nombreuses, ce qui diffère des observations in situ [16], où la largeur des réflections cristallines (et donc la taille des cristaux) semble rester inchangée au cours de la cristallisation. Cette contradiction peut s'expliquer par une orientation plus importante lors de l'étirage à mesure que la vitesse de traction augmente et ce y compris dans la phase amorphe du PET. Une proportion plus importante de matériau a pu ainsi cristalliser au cours de la relaxation [16].

\section{Conclusions}

Au cours de cette étude, nous avons mis au point et validé des outils de chauffage et de sollicitation multi-axiale. Différents trajets de chargements biaxiaux, simultanés ou séquencés ont été réalisés, les champs de déformation des ces essais hétérogènes étant mesurés par intercorrélation d'images. La microstructure des éprouvettes est alors caractérisée en terme de taux de cristallinité, d'orientation des chaînes et de tailles des cristallites, en particulier par diffraction des rayons X aux grands angles. L'influence des paramètres rhéologiques (vitesse, élongation, température) mais aussi de l'histoire du chargement, sur la microstructure du matériau est mise en évidence. Ces mesures de microstructure permettent l'étude du couplage entre orientation et cristallisation : la forte orientation résiduelle des chaînes peut s'expliquer par la cristallisation. En effet, les cristaux empêchent les chaînes de relaxer ; réciproquement, l'orientation résiduelle facilite la cristallisation des chaînes orientées de la phase amorphe.

Il est important de noter ici que la microstructure des éprouvettes après étirage n'est pas représentative de celle des bouteilles plastiques, car la microstructure du PET de la bouteille évolue aussi suivant l'épaisseur [9]. Nos essais tendent au contraire à obtenir une microstructure homogène suivant l'épaisseur de manière à découpler les effets et à identifier un modèle de comportement.

Cette étude se poursuit actuellement : d'une part des essais du même type que ceux présentés ici mais suivis 


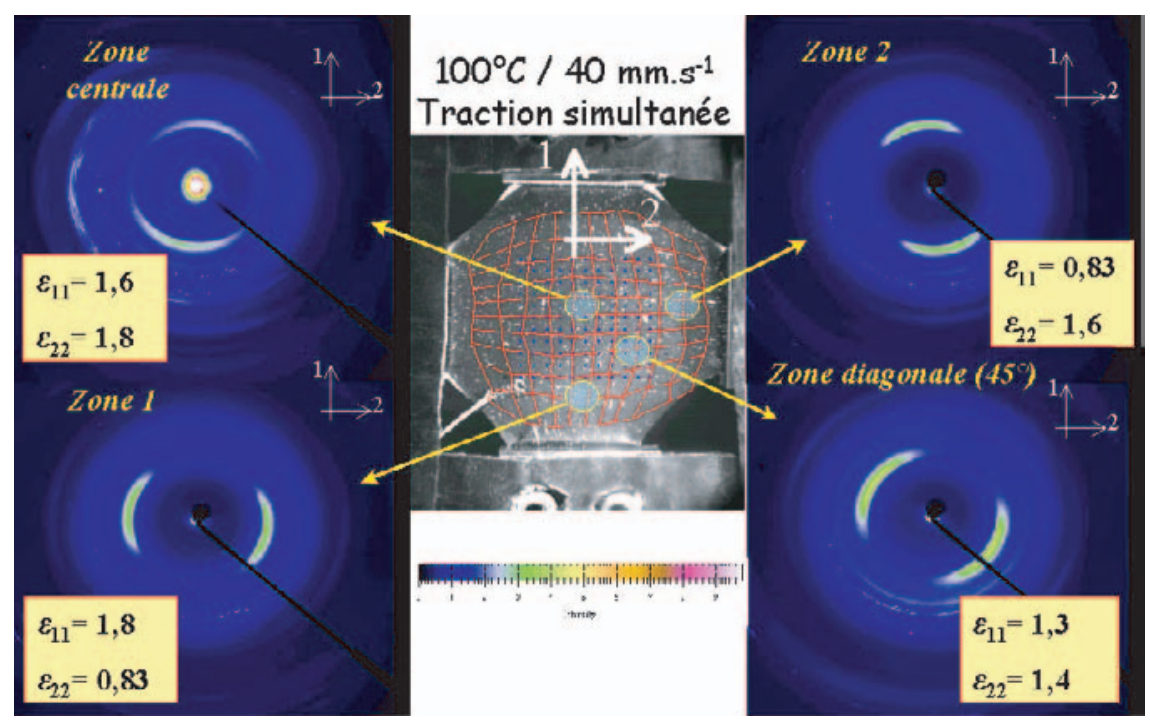

Fig. 10. Correspondance entre champ de déformation macro et orientations microscopiques.
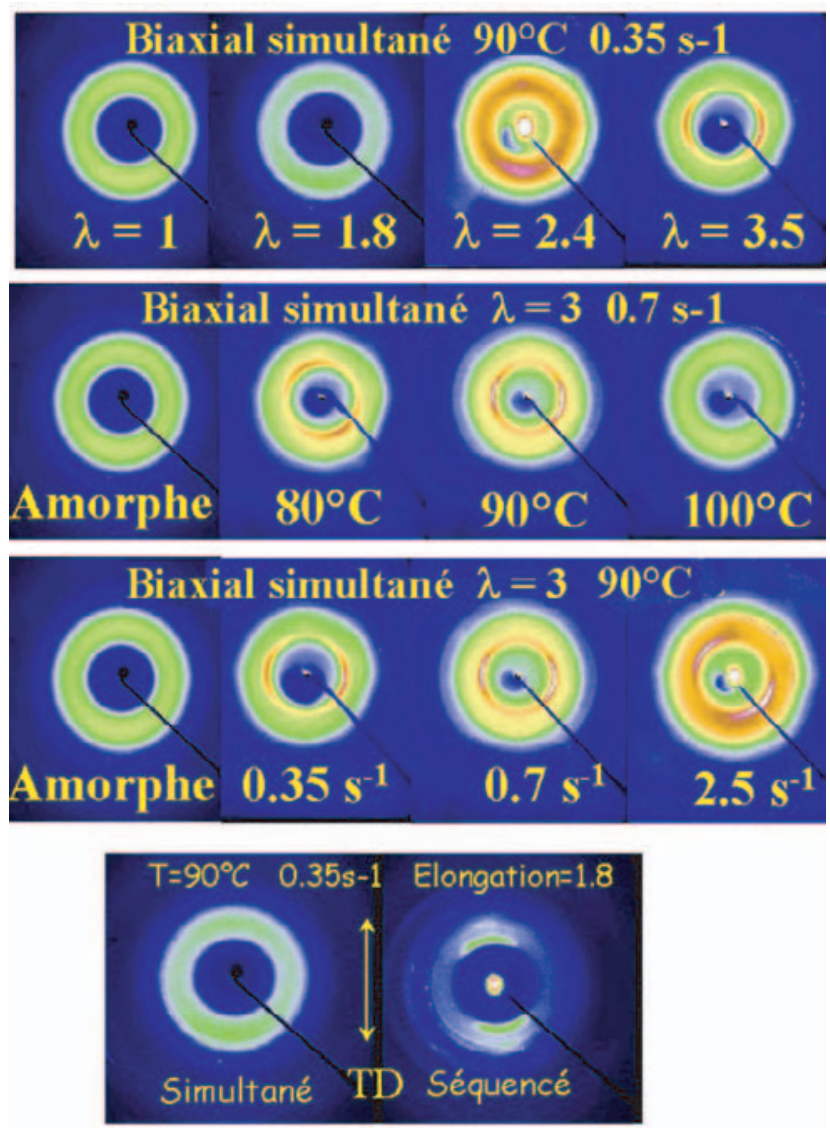

Fig. 11. Influence des paramètres sur la microstructure.

d'une trempe à l'azote liquide ont été réalisés et devraient permettre de mieux dissocier les effets de la traction et de la relaxation sur la cristallinité du matériau. Par ailleurs, des simulations numériques par éléments finis de ces essais sont en cours, de manière à identifier des modèles de comportement, par comparaison avec les résultats

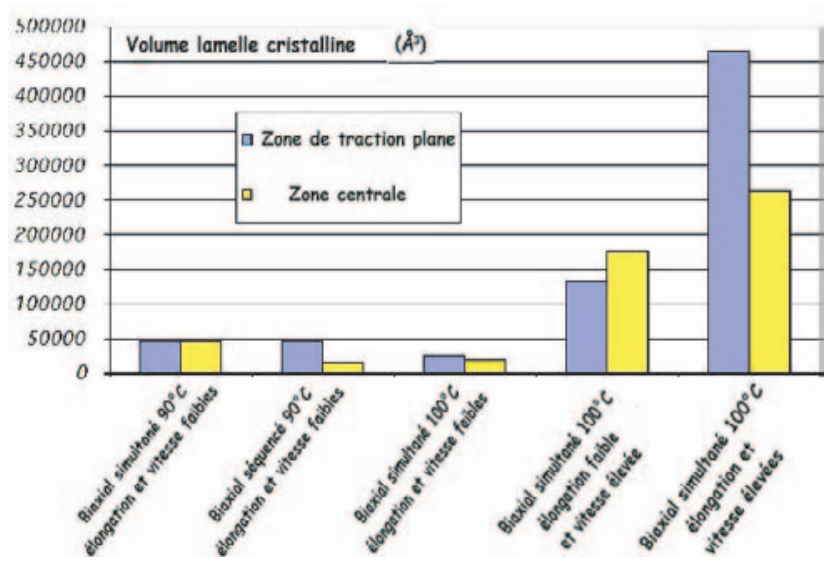

Fig. 12. Variation du volume des cristallites.

expérimentaux. Dans un premier temps, des modèles visco-élastiques utilisés classiquement pour les polymères fondus [20-22] ont été testés mais l'implantation de modèles incluant une partie hyper-élastique [23, 24] plus proche du comportement des polymères « solides » est également envisagée dans cette plage de température proche de $T g$. Le modèle adéquat pour simuler efficacement le procédé reste cependant délicat à déterminer car il doit prendre en compte non seulement la variation de microstructure et la cristallisation induite mais également la dépendance du matériau au type et à l'historique des sollicitations.

Remerciements. Nous remercions EASTMANN qui a fourni les granulés de PET et G. Régnier qui a permis l'injection des éprouvettes, ainsi que PHILIPS pour nous avoir fourni les lampes infra-rouge qui équipent le montage. Merci également à J. Doucet pour son aide pour les mesures de diffraction X. 


\section{Références}

[1] L. Chevalier, Y. Marco, G. Régnier, Mécanique \& Industries 2 (Mars 2001) 229-248

[2] M.R. Kamal, V. Tan, D.M. Kalyon, Polymer Engng. Sci. 21 (1981) 331-338

[3] M. Cakmak, J.E. Spruiell, J.L. White, J.S. Lin, Polym. Engng. Sci. 27(2) (1987) 893-905

[4] F.H. Axtell, B. Haworth, Plastic, Rubber and Composites Processing and Applications 22 (1994) 127-136

[5] M. Vigny, J.F. Tassin, A. Gibaud, G. Lorentz, Polym. Engng. Sci. 37 (1998) 1785-94

[6] M. Casey, Polymer 18 (1977) 1219-1226

[7] A. Mahendrasingam, C. Martin, W. Fuller, D.J. Blundell, D.H. MacKerron D.H., J. Oldman, J.L. Harvie, R.C. Riekel, P. Engström, Polymer 40 (1999) 5553-5565

[8] E. Gorlier, J.M. Haudin, N. Billon, Polymer 422001 9541-9549

[9] N. Everall, D. MacKerron, D. Winter, Polymer 43 (2002) 4217-4223

[10] F. Hild, B. Raka, M. Baudequin, S. Roux, F. Cantelaube, Applied Optics IP 41 (2002) 6815-6828

[11] L. Chevalier, S. Calloch, F. Hild, Y. Marco, Eur. J. Mech. A/Solids 20 (2001)169-187

[12] L. Chevalier, Y. Marco, Polym. Engng Sci. 42(2) (2002) 280-298
[13] Y. Marco, L. Chevalier, M. Chaouche, Polymer 43(24) (2002) 6569-6574

[14] R.P. Daubeny, C.W. Bunn, C.J. Brown, Proc. R. Soc. Lond. A(226) (1954) 531-542

[15] R.G. Matthews, A. Ajji, M.M. Dumoulin, R.E. Prud'homme, Polymer 41 (2000) 7139-7145

[16] A. Mahendrasingam, D.J. Blundell, C. Martin, W. Fuller, D.H. MacKerron, J.L. Harvie, R.J. Oldman, R.C. Riekel, Polymer 41 (2000) 7803-7814

[17] D.J. Blundell, A. Mahendrasingam, C. Martin, W. Fuller, D.H. MacKerron, J.L. Harvie, R.J. Oldman, C. Riekel, Polymer 41 (2000) 7793-7802

[18] M. Vigny, J.F. Tassin, G. Lorentz, Polymer 40 (1999) 397-406

[19] Y. Marco, L. Chevalier, G. Régnier, A. Poitou, Macromolecular Symposia 185 (2002) 15-34

[20] M. Doi, S.F. Edwards, J. Chem. Soc. Faraday Trans. II 74 (1975) 1789-1818

[21] A.K. Doufas, I.S. Dairanieh, A.J. McHugh, J. Rheol. 43(1) (1999) 85

[22] A. Poitou, A. Ammar, C. R. Acad. Sci. Paris t329(IIb) (2001) 5

[23] M.C. Boyce, E.M. Arruda, Polym. Engng Sci. 30 (1990) 1288

[24] C.P. Buckley, D.C. Jones, Polymer 36 (1995) 3301

Retrouvez nos articles sur le site : www.edpsciences.org/meca 